\title{
Algebraic lattices of solvably saturated formations and their applications
}

\author{
Aleksandr Tsarev and Andrei Kukharev
}

\begin{abstract}
In each group $G$ we select a system of subgroups $\tau(G)$ and say that $\tau$ is a subgroup functor if $G \in \tau(G)$ for every group $G$, and for every epimorphism $\varphi: A \rightarrow B$ and any $H \in \tau(A)$ and $T \in \tau(B)$, we have $H^{\varphi} \in$ $\tau(B)$ and $T^{\varphi^{-1}} \in \tau(A)$. We consider only subgroup functors $\tau$ such that for any group $G$ all subgroups of $\tau(G)$ are subnormal in $G$. For any set of groups $\mathfrak{X}$ the symbol $\mathrm{s}_{\tau}(\mathfrak{X})$ denotes the set of groups $H$ such that $H \in \tau(G)$ for some group $G \in \mathfrak{X}$. A formation $\mathfrak{F}$ is $\tau$-closed if $\mathrm{S}_{\tau}(\mathfrak{F})=\mathfrak{F}$. It is shown that the lattice of all $\tau$-closed totally composition formations is algebraic.
\end{abstract}

\section{Introduction}

Subgroup functors are closely related to classes of algebraic systems $[\mathbf{9}, \mathbf{1 3}]$. The concept of subgroup functor $\tau$ introduced by Skiba [13] turned out to be useful in group theory. In each group $G$ we select a system of subgroups $\tau(G)$ and say that $\tau$ is a subgroup functor if

(1) $G \in \tau(G)$ for every group $G$;

(2) for every epimorphism $\varphi: A \rightarrow B$ and any $H \in \tau(A)$ and $T \in \tau(B)$, we have $H^{\varphi} \in \tau(B)$ and $T^{\varphi^{-1}} \in \tau(A)$.

If $\tau(G)=\{G\}$ then the functor $\tau$ is called trivial. For any set of groups $\mathfrak{X}$ the symbol $\mathrm{S}_{\tau}$ denotes the set of groups $H$ such that $H \in \tau(G)$ for some group $G \in \mathfrak{X}$.

A class of groups $\mathfrak{F}$ is called $\tau$-closed if $\mathrm{s}_{\tau}(\mathfrak{F})=\mathfrak{F}$. For instance $\mathfrak{F}$ is called sclosed [6] (or hereditary) if it contains all the subgroups of $G \in \mathfrak{F}$ (i.e., $\tau(\mathfrak{F})=\mathrm{s}(\mathfrak{F})$ ), and $\mathrm{s}_{n}$-closed $[\mathbf{6}]$ (or normally hereditary) if it contains all the normal subgroups of $G \in \mathfrak{F}$ (i.e., $\tau(\mathfrak{F})=\mathrm{s}_{n}(\mathfrak{F})$ ).

Formations extend the notion of a variety of finite groups.

2010 Mathematics Subject Classification. Primary 20F17; secondary 20D10, 43A62, 20M35.

Key words and phrases. Finite group, subgroup functor, formation of groups, satellite of formation, totally composition formation, algebraic lattice of formations, formal language, hypergroup.

This work has been partially supported by the grant RSF 18-71-10007 from the Russian Science Foundation. 
Definition 1.1. A formation is a class of finite groups $\mathfrak{F}$ satisfying the following two conditions: (1) if $G \in \mathfrak{F}$, then $G / N \in \mathfrak{F}$; and (2) if $G / N_{1}, G / N_{2} \in \mathfrak{F}$, then $G / N_{1} \cap N_{2} \in \mathfrak{F}$, for any $N, N_{1}, N_{2} \unlhd G$.

A formation $\mathfrak{F}$ is called $\tau$-closed if $\tau(G) \subseteq \mathfrak{F}$ for every group $G$ of $\mathfrak{F}$. In the sequel, we will consider only subgroup functors $\tau$ such that for any group $G$ all subgroups of $\tau(G)$ are subnormal in $G$.

The theory of saturated formations introduced by Gaschütz [7] is a fundamental part of the theory of finite groups. Composition formations form a broader than saturated formations family of classes. By Baer's theorem composition formations are precisely solvably saturated formations [6, p. 373]. A formation $\mathfrak{F}$ is said to be solvably saturated [8] if it contains each group $G$ with $G / \Phi(N) \in \mathfrak{F}$ for some solvable normal subgroup $N$ of $G$, and any saturated formation is solvably saturated.

An element $a$ of a lattice $\Theta$ is compact [4] if $a \leqslant \vee\left(x_{j} \mid j \in S\right)$ holds for $a \leqslant \vee\left(x_{j} \mid j \in J\right)$ and some finite subset $S \subset J$. We recall that a complete lattice is algebraic if each its element is the union of some set of compact elements. Safonov [11] proved that the lattice of all totally saturated formations is algebraic. The lattice of all $n$-multiply composition formations is algebraic $[\mathbf{1 5}, \mathbf{2 1}]$, and the following problem was solved in $[\mathbf{1 7}]$.

Question (see [15, Problem 1]). Is the lattice of all totally composition formations algebraic?

Our theorem extends the mentioned result for $\tau$-closed classes of finite groups.

THEOREM 1.1. The lattice $c_{\infty}^{\tau}$ of all $\tau$-closed totally composition formations of finite groups is algebraic.

The present paper is organized as follows, - basic concepts are introduced in the second section, used lemmas are given in the third section, and the proof of the main result is discussed in the fourth section. In particular, it is shown that one-generated totally composition formations are compact elements of the lattice $c_{\infty}^{\tau}$. Applications of this result and some ideas for further research are presented in the last fifth section.

\section{Preliminaries}

We consider only finite groups in this paper, notations and terminologies are standard $[\mathbf{6}, \mathbf{8}, \mathbf{1 3}, \mathbf{1 5}, \mathbf{2 0}]$. If $\mathfrak{X}$ is a set of groups, then we write $(\mathfrak{X})$ to denote the intersection of all classes of groups containing $\mathfrak{X}$. In particular, (1) is the class of all identity groups. If a class of groups is a formation, it is closed with respect to forming quotient groups and subdirect products. For any group $G$ and a nonempty formation $\mathfrak{F}$, we denote by $G^{\mathfrak{F}}$ the $\mathfrak{F}$-residual of $G$, i.e., the intersection of all normal subgroups $N$ of $G$ such that $G / N \in \mathfrak{F}$; the class $\mathfrak{M F}=\left\{G \mid G^{\mathfrak{F}} \in \mathfrak{M}\right\}$ is the product of formations $\mathfrak{M}$ and $\mathfrak{F}$.

2.1. Composition formations. The set of all primes is denoted by $\mathbb{P}$. Let $p \in \mathbb{P}$, and $G$ a group. Then the subgroup $C^{p}(G)$ is the intersection of the centralizers of all the abelian $p$-chief factors of $G$ with $C^{p}(G)=G$ if $G$ has no abelian $p$-chief factors. For every set of groups $\mathfrak{X}$, we write $\operatorname{Com}(\mathfrak{X})$ to denote the class 
of all groups $L$ such that $L$ is isomorphic to some abelian composition factors of some group in $\mathfrak{X}$. If $\mathfrak{X}$ is the set of one group $G$, then we write $\operatorname{Com}(G)$ instead of $\operatorname{Com}(\mathfrak{X}) ; \pi(\mathfrak{X})$ is the set of all primes dividing the order of all groups $G \in \mathfrak{X}$. The symbol $R(G)$ denotes the product of all solvable normal subgroups of $G$. We consider a function $f$ of the form

$$
f: \mathbb{P} \cup\{0\} \rightarrow\{\text { formations of groups }\},
$$

and the class of groups

$$
C L F(f)=\left(G \mid G / R(G) \in f(0) ; G / C^{p}(G) \in f(p) \text { for all } p \in \pi(\operatorname{Com}(G))\right) .
$$

If $\mathfrak{F}$ is a formation such that $\mathfrak{F}=C L F(f)$ for a function $f$ of the form $(*)$, then $\mathfrak{F}$ is said to be composition (solvably saturated) formation, and $f$ is said to be a composition satellite of $\mathfrak{F}$; see [8, p. 4].

If the values of composition satellites of some formation are themselves composition formations, then this circumstance leads to the following natural definition. Every formation is 0-multiply composition; for $n>0$, a formation $\mathfrak{F}$ is called $n$-multiply composition if $\mathfrak{F}=C L F(f)$, and all nonempty values of $f$ are $(n-1)$ multiply composition formations; see [15]. For $n=1$ we deal with the case of composition formations. Consider an example. Let $\mathfrak{M}=\mathfrak{N}^{n} \mathfrak{H}$ and $\mathfrak{F}=\mathfrak{N}_{p} \mathfrak{M}$, where the formation $\mathfrak{H} \neq \emptyset$ is not saturated. By [13, Example 1.3.3] and [15, Corollary 4], formations $\mathfrak{M}$ and $\mathfrak{F}$ are $n$-multiply composition.

A formation is called totally composition if it is $n$-multiply composition for all positive integers $n$. The most well-known solvably saturated formations are totally composition; see [15]. In particular, the formations $\emptyset$ and (1) are totally composition.

2.2. Lattices of formations. A set of formations $\Theta$ is called a complete lattice of formations $[\mathbf{1 3}]$ if the intersection of every set of formations in $\Theta$ belongs to $\Theta$, and there is a formation $\mathfrak{F}$ in $\Theta$ such that $\mathfrak{M} \subseteq \mathfrak{F}$ for every other formation $\mathfrak{M}$ of $\Theta$. Every complete lattice of formations is a complete lattice in the ordinary sense. Various sets of formations form complete lattices; for instance the set of all saturated formations [13, p. 151], and the set of all composition (or solvably saturated) formations $[\mathbf{1 4}$, p. 97] are complete lattices of formations. Moreover for all positive integers $n$, the set of all $n$-multiply composition formations $c_{n}$, and the set of all totally composition formations $c_{\infty}=\bigcap_{n=1}^{\infty} c_{n}$ are complete lattices of formations; see [15, p. 904]. Then using [21, Lemma 3.1], we conclude that the set of all $\tau$-closed totally composition formations $c_{\infty}^{\tau}$ is a complete lattices of formations too.

A formation in $\Theta$ is called a $\Theta$-formation. Let $\Theta$ be a complete lattice of formations, and let $\left\{\mathfrak{F}_{i} \mid i \in I\right\}$ be an arbitrary set of $\Theta$-formations.

For a complete lattice $\Theta$, $\Theta$ form $\mathfrak{X}$ is the intersection of all $\Theta$-formations containing a set of groups $\mathfrak{X}$, and $c_{\infty}^{\tau}$ form $\mathfrak{X}$ is the intersection of all $\tau$-closed totally composition formations containing a set of groups $\mathfrak{X}$. We denote $\bigvee_{\Theta}\left(\mathfrak{F}_{i} \mid i \in I\right)=$ $\Theta$ form $\left(\bigcup_{i \in I} \mathfrak{F}_{i}\right)$. In particular, we write $\bigvee_{\infty}^{\tau}\left(\mathfrak{F}_{i} \mid i \in I\right)=c_{\infty}^{\tau}$ form $\left(\bigcup_{i \in I} \mathfrak{F}_{i}\right)$. 
If $\mathfrak{M}, \mathfrak{H} \in \Theta$, then $\mathfrak{M} \bigcap \mathfrak{H}$ is the greatest lower bound for $\{\mathfrak{M}, \mathfrak{H}\}$ in $\Theta$; and $\mathfrak{M} \bigvee_{\Theta} \mathfrak{H}$ is the least upper bound for $\{\mathfrak{M}, \mathfrak{H}\}$ in $\Theta$.

Let $\left\{f_{i} \mid i \in I\right\}$ be a set of $\Theta$-valued functions of the form $(*)$. Then by $\bigvee_{\Theta}\left(f_{i} \mid i \in I\right)$ we denote a function $f$ such that $f(a)=\Theta$ form $\left(\bigcup_{i \in I} f_{i}(a)\right)$ for all $a \in \mathbb{P} \cup\{0\}$.

\section{Lemmata}

Lemma 3.1. [15, Lemma 2] Let $\mathfrak{F}=\bigcap_{i \in I} \mathfrak{F}_{i}$, where $\mathfrak{F}_{i}=C L F\left(f_{i}\right)$. Then $\mathfrak{F}=C L F(f)$, where $f=\bigcap_{i \in I} f_{i}$.

Let $\left\{f_{i} \mid i \in I\right\}$ be the set of all composition $c_{\infty}^{\tau}$-valued satellites of a formation $\mathfrak{F}$. Since the lattice $c_{\infty}^{\tau}$ is complete, using Lemma 3.1, we conclude that $f=\bigcap_{i \in I} f_{i}$ is a composition $c_{\infty}^{\tau}$-valued satellite of $\mathfrak{F}$. The satellite $f$ is called minimal.

LEMMA 3.2. [19] Let $\mathfrak{X}$ be a nonempty set of groups, $\mathfrak{F}=c_{\infty}^{\tau}$ form $\mathfrak{X}$, and $\pi=\pi(\operatorname{Com}(\mathfrak{X}))$, and let $f$ be the minimal $c_{\infty}^{\tau}$-valued composition satellite of $\mathfrak{F}$. Then the following statements hold:

1) $f(0)=c_{\infty}^{\tau}$ form $(G / R(G) \mid G \in \mathfrak{X})$;

2) $f(p)=c_{\infty}^{\tau}$ form $\left(G / C^{p}(G) \mid G \in \mathfrak{X}\right)$ for all $p \in \pi$;

3) $f(p)=\varnothing$ for all $p \in \mathbb{P} \backslash \pi$;

4) if $\mathfrak{F}=C L F(h)$ and the satellite $h$ is $c_{\infty}^{\tau}$-valued, then for all $p \in \pi$ we have

$$
\begin{gathered}
f(p)=c_{\infty}^{\tau} \operatorname{form}\left(G \mid G \in h(p) \cap \mathfrak{F} \text { and } O_{p}(G)=1\right), \text { and } \\
f(0)=c_{\infty}^{\tau} \operatorname{form}(G \mid G \in h(0) \cap \mathfrak{F} \text { and } R(G)=1) .
\end{gathered}
$$

A group $G$ is monolithic if it has a unique minimal normal subgroup (a monolith), and this is contained in every nontrivial normal subgroup. The socle $\operatorname{Soc}(G)$ of a group $G \neq 1$ is the product of all minimal normal subgroups of $G$.

Lemma 3.3. [19, Lemma 9] Let $A$ be a monolithic group with a nonabelian socle $R, \mathfrak{M}$ a semiformation and $A \in c_{n}^{\tau}$ form $\mathfrak{M}, n \geqslant 0$. Then $A \in \mathfrak{M}$.

Recall that a semiformation is a class of groups closed under taking homomorphic images.

LEMMA 3.4. [8, Lemma 1.3, p. 250] Let $Z_{p}$ be a group of a prime order $p$, and $G$ be a group with $O_{p}(G)=1$. Suppose that $T=Z_{p} \imath G=[K] G$ is the regular wreath product, where $K$ is the base group of $T$. Then $K=C^{p}(T)=O_{p}(T)$.

LEMMA 3.5. [15, Lemma 4] Let $\mathfrak{F}=C L F(f)$. If $G / O_{p}(G) \in f(p) \cap \mathfrak{F}$ for some prime $p$, then $G \in \mathfrak{F}$.

The following lemma is proved by direct calculation.

LEMMA 3.6. Let $f_{i}$ be the minimal $c_{\infty}^{\tau}$-valued composition satellite of a formation $\mathfrak{F}_{i}$, where $i \in I$. Then $f=\bigvee_{\infty}^{\tau}\left(f_{i} \mid i \in I\right)$ is the minimal $c_{\infty}$-valued composition satellite of formation $\mathfrak{F}=\bigvee_{\infty}^{\tau}\left(\mathfrak{F}_{i} \mid i \in I\right)$.

One of the most elementary nontrivial examples of saturated formation is the class $\mathfrak{S}_{\pi}$ of all solvable $\pi$-groups. (We note that $\mathfrak{S}_{\emptyset}=(1)$.) The following lemma we obtain by $[\mathbf{1 5}$, Theorem 6] and the proof of [12, Lemma 12]. 
Lemma 3.7. Let $\mathfrak{F}$ be a nonempty $\tau$-closed formation. Then $\mathfrak{S}_{\pi} \mathfrak{F}$ is a $\tau$-closed totally composition formation, where $\pi(\mathfrak{F}) \subseteq \pi \subseteq \mathbb{P}$.

Lemma 3.8. Let $\mathfrak{F}=\bigvee_{\infty}^{\tau}\left(\mathfrak{F}_{i} \mid i \in I\right)$, where $\mathfrak{F}_{i} \in c_{\infty}^{\tau}$ for all $i \in I$, and let $A$ be a monolithic $\mathfrak{F}$-group with a nonabelian socle $R$. Then $A \in \bigcup_{i \in I} \mathfrak{F}_{i}$.

Proof. By Lemma 3.7, we obtain that $\mathfrak{F} \subseteq \mathfrak{M}=\mathfrak{S}_{\pi} c_{0}^{\tau}$ form $\left(\bigcup_{i \in I} \mathfrak{F}_{i}\right)$, where $\pi=\pi(\mathfrak{F})$. Then $A \in \mathfrak{M}$. Moreover, $A \in c_{0}^{\tau}$ form $\left(\bigcup_{i \in I} \mathfrak{F}_{i}\right)$, since $R=\operatorname{Soc}(A)$ is a nonabelian group, and $A$ belongs to $\bigcup_{i \in I} \mathfrak{F}_{i}$ by Lemma 3.3 .

\section{Compact elements}

Proposition 4.1. For any group $G$, the one-generated totally composition formation $\mathfrak{F}=c_{\infty}^{\tau}$ form $G$ is a compact element of the lattice $c_{\infty}^{\tau}$.

Proof. We shall use induction on $|G|$; let $A$ be a counterexample of the minimal order, and $\mathfrak{F}=c_{\infty}^{\tau}$ form $A \subseteq \mathfrak{M}=c_{\infty}^{\tau}$ form $\left(\bigcup_{i \in I} \mathfrak{F}_{i}\right)=\bigvee_{\infty}^{\tau}\left(\mathfrak{F}_{i} \mid i \in I\right)$, where $\mathfrak{F}_{i} \in c_{\infty}^{\tau}$ for all $i \in I$.

(i) We shall show that $A$ is a monolithic group. Suppose that $M_{1} \neq M_{2}$ are two its minimal normal subgroups; and let $\mathfrak{M}_{j}=c_{\infty}^{\tau}$ form $\left(A / M_{j}\right)$, where $j=1,2$. We have $\left|A / M_{j}\right|<|A|$, so by induction we have $\mathfrak{M}_{j} \subseteq \mathfrak{M}$, which gives

$$
\mathfrak{M}_{1} \subseteq c_{\infty}^{\tau} \text { form }\left(\mathfrak{F}_{i_{1}} \bigcup \ldots \bigcup \mathfrak{F}_{i_{t}}\right) \text {, and } \mathfrak{M}_{2} \subseteq c_{\infty}^{\tau} \text { form }\left(\mathfrak{F}_{i_{t+1}} \bigcup \ldots \bigcup \mathfrak{F}_{i_{s}}\right)
$$

for some $i_{1}, \ldots, i_{s}$.

Thus $\mathfrak{F}=\mathfrak{M}_{1} \bigvee_{\infty}^{\tau} \mathfrak{M}_{2} \subseteq c_{\infty}^{\tau}$ form $\left(\mathfrak{F}_{i_{1}} \bigcup \ldots \bigcup \mathfrak{F}_{i_{t}} \cup \mathfrak{F}_{i_{t+1}} \cup \ldots \cup \mathfrak{F}_{i_{s}}\right)$, which is a contradiction.

(ii) Let $R=\operatorname{Soc}(A)$. If $R$ is a nonabelian group, then by Lemma 3.8, we have $A \in \bigcup_{i \in I} \mathfrak{F}_{i}$, since $A \in \mathfrak{M}$. This is a contradiction.

(iii) Thus, $R$ is an abelian $p$-group for some prime $p \in \pi(\operatorname{Com}(A))$. Since $A / \Phi(A) \in$ form $A$, we have $c_{\infty}^{\tau}$ form $(A / \Phi(A))=c_{\infty}^{\tau}$ form $A$; and since $|A / \Phi(A)|<$ $|A|$, by induction, we conclude that $R \nsubseteq \Phi(A)$. Let $B$ be some subgroup of $A$ with $R \cap B=1$ and $O_{p}(B)=1$. Then $A=Z_{p} \curlywedge B=[R] B$; and, by lemma 3.4, we have $R=C^{p}(A)=O_{p}(A)$. Let $f_{i}, f$, and $m$ be minimal $c_{\infty}^{\tau}$-valued composition satellites of formations $\mathfrak{F}, \mathfrak{F}_{i}$, and $\mathfrak{M}$, respectively. Lemma 3.6 implies $m=\bigvee_{\infty}^{\tau}\left(f_{i} \mid i \in I\right)$. Applying the properties of regular wreath products, we have

$$
B \cong A / O_{p}(A)=A / R=A / C^{p}(A) \in m(p) .
$$

Since $|B|<|A|$, by induction, we can find a set $J=\left\{j_{1}, j_{2}, \ldots, j_{k}\right\} \subseteq I$ such that $B \cong A / C^{p}(A) \in f_{j_{1}}(p) \bigvee_{\infty}^{\tau} \ldots \bigvee_{\infty}^{\tau} f_{j_{k}}(p)$. By lemma 3.6, $m_{3}=\bigvee_{\infty}^{\tau}\left(f_{j} \mid j \in J\right)$ is the minimal $c_{\infty}^{\tau}$-valued composition satellites of $\mathfrak{M}_{3}=\bigvee_{\infty}^{\tau}\left(\mathfrak{F}_{j} \mid j \in J\right)$. Thus, $A / O_{p}(A) \cong B \in m_{3}(p)$; and finally by Lemma 3.5 , we have $A \in \mathfrak{M}_{3}$. That implies $\mathfrak{F}=c_{\infty}^{\tau}$ form $A \subseteq \mathfrak{M}_{3}$, contradicting the above.

Proof of THE THEOREM. Let $\mathfrak{F}$ be a $\tau$-closed totally composition formation. Obvious we have $\mathfrak{F}=c_{\infty}^{\tau}$ form $\left(\bigcup_{i \in I} \mathfrak{F}_{i}\right)=\bigvee_{\infty}^{\tau}\left(\mathfrak{F}_{i} \mid i \in I\right)$, where $\mathfrak{F}_{i}=c_{\infty}^{\tau}$ form $G_{i}$ for some finite group $G_{i}$ and $i \in I$. Therefore, it suffice to show that each onegenerated formation $\mathfrak{F}_{i}$ is a compact element of the lattice $c_{\infty}^{\tau}$. This follows by Proposition 5.1. 
COROLlary 4.1. [17] The lattice of all totally composition formations of finite groups is algebraic.

\section{Final remarks}

5.1. Inductive lattices of formations. Skiba [13] introduced the concept of an inductive lattice of formations in order to adapt lattice-theoretical methods for the investigation of saturated formations. This concept plays an important role in the research of the lattices of formations and their law systems (see Chapter 4 of the book [13], Chapter 4 of the book [20]). Both the lattice of all totally saturated formations $[\mathbf{2 2}]$ and the lattice of all $\tau$-closed totally composition formations $[\mathbf{1 6}]$ are inductive.

Let $\Theta$ be a complete lattice of formations. A satellite $f$ is called $\Theta$-valued if all its values belong to $\Theta$. We denote by $\Theta^{c}$ the set of all formations having a composition $\Theta$-valued satellite. In [15, p. 901], it is shown that this set is a complete lattice of formations. A complete lattice $\Theta^{c}$ is called inductive if for any collection of formations $\left\{\mathfrak{F}_{i}=C L F\left(f_{i}\right) \mid i \in I\right\}$, where $f_{i}$ is an integrated satellite of $\mathfrak{F}_{i} \in \Theta^{c}$, the following equality holds: $\bigvee_{\Theta^{c}}\left(\mathfrak{F}_{i} \mid i \in I\right)=C L F\left(\bigvee_{\Theta}\left(f_{i} \mid i \in I\right)\right)$. The inductance of a lattice $\Theta^{c}$, in fact, means that a research of the operation $\bigvee_{\Theta^{c}}$ on the set $\Theta^{c}$ can be reduced to a research of the operation $\bigvee_{\Theta}$ on the set $\Theta$. Therefore, the inductance is one a very powerful property of the lattice $\Theta^{c}$.

By Theorem 1.1, every composition formation is the join of some one-generated composition formations, and the inductance of the lattice $c_{\infty}^{\tau}$ implies the following result.

Proposition 5.1. For any groups $G_{i}$ and formations

$$
\mathfrak{F}_{i}=C L F\left(f_{i}\right)=c_{\infty}^{\tau} \text { form } G_{i},
$$

where $i \in I$, we have

$$
\bigvee_{\infty}^{\tau}\left(\mathfrak{F}_{i} \mid i \in I\right)=C L F\left(\bigvee_{\infty}^{\tau}\left(f_{i} \mid i \in I\right)\right)
$$

5.2. Formations of group languages. Languages are subsets of a certain type of monoid, the free monoid over an alphabet. Regular languages are precisely the behaviours of finite automata. A language is regular if its syntactic monoid is a finite monoid (a regular language is a group language if its syntactic monoid is a finite group). By $A^{*}$ we denote a free monoid on a set $A$, i.e., the set of all words with letters from $A$. A class of regular languages $\mathcal{C}$ associates with each finite alphabet $A$ a set $\mathcal{C}\left(A^{*}\right)$ of regular languages of $A^{*}$.

We consider only finite monoids later on. A formation of languages $[2]$ is a class of regular languages $\mathcal{F}$ satisfying the following two conditions: (1) for each alphabet $A, \mathcal{F}\left(A^{*}\right)$ is closed under Boolean operations and quotients, and (2) if $L$ is a language of $\mathcal{F}\left(B^{*}\right)$ and $\eta: B^{*} \rightarrow M$ denotes its syntactic morphism, then for each monoid morphism $\alpha: A^{*} \rightarrow B^{*}$ such that $\eta \circ \alpha$ is surjective, the language $\alpha^{-1}(L)$ belongs to $\mathcal{F}\left(A^{*}\right)$.

Following [2], we associate with each formation of monoids $\mathfrak{M}$ the class of languages $\mathcal{F}(\mathfrak{M})$ as follows; for each alphabet $A$, by $\mathcal{F}(\mathfrak{M})\left(A^{*}\right)$ we denote the set of 
languages of $A^{*}$ fully recognised by some monoid of $\mathfrak{M}$ (or, equivalently, whose syntactic monoid belongs to $\mathfrak{M}$ ). Given a formation of languages $\mathcal{F}$. We denote by $\mathfrak{M}(\mathcal{F})$ the formation of monoids generated by the syntactic monoids of the languages of $\mathcal{F}$. By the Formation Theorem [2], the correspondences $\mathfrak{M} \rightarrow \mathcal{F}(\mathfrak{M})$ and $\mathcal{F} \rightarrow \mathfrak{M}(\mathcal{F})$ are two mutually inverse, order preserving, bijections between formations of monoids and formations of languages. In particular we have a one-to-one correspondence between one-generated composition formations of finite groups and some formations of languages. Languages corresponding to saturated formations of finite groups were studien in $[\mathbf{3}, \mathbf{1 8}]$. Naturally rises the following question: How to describe the languages corresponding to (one-generated) $\tau$-closed totally composition formations of finite groups?

5.3. Classes of hypergroups. The concept of formation appeared first in the 1960s in connection with finite solvable groups. Further research showed that formations are of general algebraic nature and can be applied to the study of not necessarily solvable finite and infinite groups, Lie algebras, universal algebras and even of a general algebraic system.

In 1934, at the eight Congress of Scandinavian Mathematics, Marty [10] introduces a concept of algebraic hyperstructure, which naturally generalizes classical algebraic structures such as groups and rings. As mentioned in [5], the first example of hypergroups, which motivates the introduction of this structure, is the quotioent of a finite group by arbitrary (not necessary normal) subgroup, i.e., if the subgroup is not normal, then the quotient is not a group, but it is always a hypergroup with respect to a certain hyperoperation. Keeping in mind this idea, we can introduce a concept of hyperformation, assuming that subgroups in Definition 1.1 are not necessarily normal.

DeFinition 5.1. A hyperformation is a class of hypergroups $\mathfrak{F}$ satisfying the following two conditions: (1) if $H \in \mathfrak{F}$, then $H / N \in \mathfrak{F}$; and (2) if $H / N_{1}, H / N_{2} \in \mathfrak{F}$, then $H / N_{1} \cap N_{2} \in \mathfrak{F}$, for any subhypergroups $N, N_{1}, N_{2}$ of $H$.

It will be interesting to study the relation between classical one-generated (saturated, composition) formations and hyperformations. Finally, we note that recently in [1], some examples of hypergroups associated with some models of biological inheritance were considered.

\section{References}

[1] M. Al Tahan, B. Davvaz, A New Relationship Between Intuitionistic Fuzzy Sets and Genetics, J. Classif. (2018). https://doi.org/10.1007/s00357-018-9276-8

[2] A. Ballester-Bolinches, J.-É. Pin, X. Soler-Escrivà, Formations of finite monoids and formal languages: Eilenberg's variety theorem revisited, Forum Math. 26 (6) (2014) 1737-1761.

[3] A. Ballester-Bolinches, J.-É. Pin, X. Soler-Escrivà, Languages associated with saturated formations of groups, Forum Math. 27 (3) (2015) 1471-1505.

[4] G.D. Birkhoff, Lattice Theory, Am. Math. Soc. (1979).

[5] B. Davvaz, I. Cristea, Fuzzy algebraic hyperstructures. Studies in Fuzziness and soft computing, (2015) 321.

[6] K. Doerk, T. Hawkes, Finite Soluble Groups, De Gruyter Expositions in Mathematics, 4, Walter de Gruyter, Berlin, New York, 1992. 
[7] W. Gaschütz, Zur Theorie der endlichen auflösbaren Gruppen, Mathematische Zeitschrift, 80, no. 4, 1963, pp. 300-305.

[8] W. Guo, Structure Theory for Canonical Classes of Finite Groups, Springer-Verlag Berlin Heidelberg, 2015, 359 p.

[9] S.F. Kamornikov, M.V. Selkin, Subgroup Functors and Classes of Finite Groups, Belaruskaya Navuka, Minsk (2003) 254 p. (in Russian).

[10] F. Marty, Sur une generalization de la notion de groupe, 8th Congress Math. Scandenaves, Stockholm, (1934) 45-49.

[11] V.G. Safonov, The property of being algebraic for the lattice of all $\tau$-closed totally saturated formations, Algebra and Logic 45, no. 5 (2006), pp. 353-356.

[12] V.G. Safonov, Characterization of the soluble one-generated totally saturated formations of finite groups, Siberian Mathematical Journal 48, no. 1 (2007), pp. 150-155.

[13] A.N. Skiba, Algebra of Formations, Belaruskaya Navuka, Minsk, 1997, 240 p. (in Russian).

[14] L.A. Shemetkov, A.N. Skiba, Formations of Algebraic Systems. Sovremennaya Algebra, Nauka, Moscow, 1989, 256 p. (in Russian).

[15] A.N. Skiba, L.A. Shemetkov, Multiply $\mathfrak{L}$-composition formations of finite groups, Ukrainian Math. Journal 52, no. 6, 2000, pp. 898-913.

[16] A. Tsarev, Inductive lattices of totally composition formations, Revista Colombiana de Matemáticas 52, no. 2, 2018, pp. 161-169.

[17] A. Tsarev, On the lattice of all totally composition formations of finite groups, Ricerche mat (2019). https://doi.org/10.1007/s11587-019-00433-3.

[18] A. Tsarev, Formations of finite monoids and their applications: formations of languages and $\tau$-closed saturated formations of finite groups, Ann Univ Ferrara (2019). https://doi.org/10.1007/s11565-019-00316-3

[19] A.A. Tsarev, N.N. Vorob'ev, On a question of the theory of partially composition formations, Algebra Colloq. 21, no. 3, 2014, pp. 437-447.

[20] N.N. Vorob'ev, Algebra of Classes of Finite Groups, P.M. Masherov Vitebsk State University, Vitebsk, 2012, 322 p. (in Russian).

[21] N.N. Vorob'ev, A.A. Tsarev, On the modularity of a lattice of $\tau$-closed n-multiply $\omega$ composition formations, Ukrainian Math. Journal, 62, no. 4, 2010, pp. 453-463.

[22] N.N. Vorob'ev, On one question of the theory of local classes of finite groups, Problems in Algebra Proc. F. Scorina Gomel State Univ., Gomel. 14, 1999, pp. 132-140.

Department of Mathematics, Jeju National University, 690-756, Jeju, South Korea

Department of Mathematics \& IT, P.M. Masherov Vitebsk State University, 33 Moscow Avenue, 210038, Vitebsk, Belarus

E-mail address: alex_vitebsk@mail.ru (corresponding author)

Siberian Federal University, 79 Svobodny Avenue, 660041, Krasnoyarsk, Russia

Department of Mathematics \& IT, P.M. Masherov Vitebsk State University, 33 Moscow Avenue, 210038, Vitebsk, Belarus

E-mail address: kukharev.av@mail.ru 